\title{
Neck Lymph Node Boundary Sublevel IB
}

National Cancer Institute

\section{Source}

National Cancer Institute. Neck Lymph Node Boundary Sublevel IB. NCI Thesaurus. Code C132496.

The following anatomical structures define the neck lymph node boundary for sublevel IB: Superior: body of the mandible; inferior: posterior belly of the digastric muscle; anterior (medial): anterior belly of the digastric muscle; posterior (lateral): stylohyoid muscle. (AJCC 8th ed.) 